\title{
SISTEMAS DE REPRESENTACIÓN EN LA ENSEÑANZA DE LOS PUNTOS CRÍTICOS: PERSPECTIVA HISTÓRICA
}

\author{
Sistemas de representação no ensino dos \\ pontos críticos: perspectiva bistórica
}

Maria Teresa González Astudillo ${ }^{1}$

\section{Resumen}

El análisis de libros de texto aporta gran información tanto acerca de las concepciones en relación con el contenido matemático que desarrollan, como acerca del proceso educativo con el que están relacionados. Por ello se ha desarrollado un modelo de análisis de libros de texto que permite caracterizar la información, estructura y forma de hacer matemáticas en cada momento histórico. Dicho modelo se desarrolla en tres etapas con diferente nivel de profundidad y especificidad. En el primer nivel se elabora la ficha de referencia de cada texto que permite identificarlo. El segundo nivel consiste en un estudio global para ubicar la obra en el momento histórico y científico en el que se gestó su nacimiento. En el tercer nivel se trata de estudiar los tipos de representación que aparecen en cada uno de los libros. El modelo diseñado se ha aplicado a diferentes libros de Análisis Matemático a partir del primero que fue escrito en el año 1696, y considerando las más relevantes publicaciones realizadas en el siglo XVIII.

Palabras clave: Historia de la educación matemática; Libros de texto; Análisis matemático.

Doctora por la Universidad e Salamanca Profesora Titular del Departamento de Didáctica de las Matemáticas y las Ciencias Experimentales de la Universidad de Salamanca (España)// maite@usal.es 


\section{Resumo}

A análise de livros de texto fornece muita informação tanto acerca das concepções em relação ao conteúdo matemático que desenvolvem como acerca do processo educativo com o que estão relacionados. Por esse motivo, desenvolveu-se um modelo de análise de livros de texto que permite caracterizar a informação, estrutura e forma de fazer matemática em cada momento histórico. Esse modelo desenvolve-se em três etapas, com um nível diferente de profundidade e especificidade. No primeiro nível, elabora-se a ficha de referência de cada texto que permite fazer a sua identificação. $O$ segundo nível consiste num estudo global para enquadrar a obra no momento histórico e científico em que se deu o seu nascimento. No terceiro nível, estudam-se os tipos de representação que aparecem em cada um dos livros. O modelo desenhado aplicou-se a diferentes livros de Análise Matemática a partir do primeiro que foi escrito no ano de 1696 e considerada a publicação mais relevante realizada no século XVIII .

Palavras-chave: História da Educação Matemática; Livros Didáticos; Análise Matemática

\section{Introducción}

El propósito de este trabajo ha sido realizar un estudio histórico en relación con la enseñanza de los puntos críticos partiendo de su introducción en el siglo XVII e intentando responder a los siguientes interrogantes:

- ¿Qué características poseen cada uno de los sistemas simbólicos de representación utilizados en el Análisis Matemático?

- ¿Qué tipo de sistemas simbólicos de representación utilizaban los matemáticos de los siglos XVII y XVIII en relación con los puntos críticos y de qué forma?

Los objetivos principales fueron:

- Identificar y caracterizar los diferentes sistemas simbólicos de representación en el campo del Análisis Matemático.

- Analizar los cambios producidos en dichos sistemas de representación desde su origen, estableciendo diferentes etapas en su evolución, buscando las causas.

- Describir cada uno de los libros analizados en función de los sistemas simbólicos de representación utilizados.

Las diferentes etapas del trabajo incluyen: una labor de recopilación de las investigaciones previas sobre el tema, la localización de las fuentes 
documentales y selección del material a analizar, el establecimiento de las categorías de análisis que constituirían la metodología que se iba a utilizar, la recogida de datos que permitió cubrir los objetivos propuestos y finalmente el establecimiento de resultados, conclusiones y perspectivas para el futuro.

\section{Materiales y método}

Esta investigación ${ }^{2}$ se enmarca en la línea de investigación de Historia de la Educación Matemática que según Fox (1981), es una labor útil y actual caracterizada por tratar de aclarar problemas de interés actual mediante un estudio intensivo de materiales ya existentes. Concretando un poco más, Cohen y Manion (1990) la describen indicando que se refiere a

la situación, evaluación y síntesis de la evidencia sistemática y objetiva con el fin de establecer los hechos y extraer las conclusiones acerca de acontecimientos pasados". El pasado "además incluye no sólo hechos y acontecimientos, sino también estructuras y procesos, sincronías y diacronías de distinta naturaleza y espesor (VIÑAO, 1997, p. 27).

En este sentido es interesante considerar la investigación histórica como un proceso de búsqueda sistemática de datos que respondan preguntas acerca de fenómenos del pasado con el propósito de alcanzar una mejor comprensión de instituciones, prácticas, tendencias y en nuestro caso aspectos relacionados con la educación (GALL et al, 1996). Por ello partimos de que: "el análisis histórico cumple varias funciones: en primer lugar pone de manifiesto que las nociones matemáticas no se han desarrollado de manera aislada, sino conectadas unas con otras; en segundo lugar, muestra el contexto de problemas en los que han aparecido los conceptos, y en tercer lugar, nos hace comprender que el desarrollo no ha sido lineal, sino con avances, retrocesos e indecisiones, nos da cuenta, en definitiva, de las concepciones que han aparecido a lo largo de la historia de la matemática" (SIERRA, GONZÁLEZ; LÓPEZ, 2002).

En este sentido se están desarrollando en la Universidad de Salamanca diversas investigaciones que tiene en común el método de investigación histórico caracterizado por las siguientes fases: Planteamiento de la investigación, Heurística. Crítica, Análisis de la documentación, Hermeneútica y Exposición.

2 Esta investigación se presentó en el Encontro Internacional em Història da Educação Matemática que se celebró en Porto/Portugal el día 16 de julio de 2005. 
En concreto la investigación que se expone a continuación trata de responder a la pregunta ¿qué tipos de sistemas simbólicos utilizaban los matemáticos de los siglos XVII, XVIII y XIX en relación con los puntos críticos y de qué forma? Para ello se seleccionaron algunas fuentes primarias que permitieron descubrir las primeras incorporaciones de los sistemas de representación simbólicos al Análisis Matemático.

Para poder realizar esta investigación primero se realizó un estudio histórico acerca de esta rama de las matemáticas. Las fuentes que se utilizaron han sido fundamentalmente libros de historia de la Matemática: Boyer (1986), Durán (1996), Dhombres y otros (1987), Duham (1990), Grattan-Guinness (1984), Hairer y Wanner (1996), Wussing (1998); artículos en los que se trata la contribución de los matemáticos al cálculo diferencial: Cantoral (1995), Dhombres (1985), Schubring (1987) y libros universitarios de cálculo diferencial: Apóstol (1981) y Bradley y Smith (1998).

A partir de la información obtenida, se elaboró un primer listado de los libros publicados en relación con el Análisis Matemático. Después de esta primera aproximación, se realizó un registro y localización de dichos libros y finalmente una selección adecuada. Los criterios para efectuar la selección de los textos de matemáticas que iban a ser objeto de estudio han sido los siguientes:

- Que los textos cumpliesen la función de libro de texto o fuesen escritos con este propósito.

- Que correspondieran a diferentes momentos de la enseñanza del Análisis Matemático para poder estudiar la evolución de dichas representaciones.

- Que los textos contuvieran conceptos relativos a los puntos críticos.

De esta forma, hemos cubierto un periodo de 150 años en la historia de la Matemática, siendo los principales textos franceses, salvo las excepciones correspondientes a un libro inglés, otro alemán aunque escrito en latín y otro español. Los libros seleccionados han sido:

- L’Hôpital, G.F.A. (1696) Análisis des infiniment petits. París: L'Imprimierie Royale.

- MacLaurin, C. (1742) Traité des fluxions. París: Quay des Augustius

- Euler, L. (1755) Institutiones calculi differentialis. San Petersburgo: Academia Imperialis Scientriaum.

- Bézout, E. (1764) Cours de Mathematiques. París: De l'Imprimerie de Ph- D. Pierres.h 
- Bails, B. (1797) Principios de matemáticas. Madrid: Imprenta de la viuda de Joaquín Ibarra

- Lagrange, J. L. (1797) Theorie des fonctions analytiques. París: L'Imprimerie de la Republique.

- Lacroix, S. (1837) Traité elementaire de calcul différentiel et calcul intégral. París: Gauthiers-Villars

Para realizar el análisis de estos libros se realizó, en primer lugar, un vaciado de diferentes investigaciones relacionadas con el análisis de libros de texto buscando el instrumento idóneo que nos permitiera ir recogiendo datos y analizándolos para ir obteniendo resultados en torno a los objetivos fijados. Esta búsqueda se llevó a cabo en dos frentes, por un lado se analizaron las investigaciones de los historiadores de la educación en relación con el análisis de libros de texto: Choppin (1993), y por otro, estudiamos aportaciones dentro del campo de la educación matemática: el estudio comparativo que ha realizado Howson (1995) con libros de texto de diferentes países; los trabajos de Chevallard (1985), Chevallard y Joshua (1982) y Tavignot (1993) en relación con la transposición didáctica; la investigación de Pimm $(1987,1994)$ sobre la legibilidad de los textos matemáticos; el estudio de Otte (1986) sobre las interpretaciones de los textos; la clasificación de Dormolen (1986) de los elementos imprescindibles que debe tener un libro de texto y las consideraciones de Lowe y Pimm (1996) acerca de las interacciones entre el lector, el escritor, el profesor y el libro en el aula de matemáticas.

El análisis de los libros de texto se realizó en tres etapas con diferentes niveles de profundización:

En primer lugar, se rellenó una ficha de referencia de cada una de las obras objeto de análisis, en la que se incluye: autor, año de nacimiento y fallecimiento, título de la obra, año de la primera edición indicando editorial, año de la edición consultada incluyendo editorial y lugar de edición, y localización del manual consultado.

Seguidamente se analizó la obra desde un punto de vista general, relacionándola con el contexto en el que se ha generado dicho libro de texto. Este apartado nos dio información general acerca del texto, contextualizándolo para poder analizarlo correctamente en función de las circunstancias que rodearon su publicación. En él se reflejaron las circunstancias que hicieron que fuera escrito del modo en que ha llegado a nosotros, los objetivos que pretendía el autor, las innovaciones que se introdujeron, así como la estructura que posee el libro, es decir, su extensión, la secuenciación de los contenidos, las características de impresión o de presentación...

Finalmente, se realizó un estudio de los diferentes sistemas de representación simbólicos en Análisis Matemático para lo que se utilizó la clasifica- 
ción realizada por Janvier (1987) de dichos sistemas. De cada uno de los textos se han seleccionado sucesivas unidades de información, que se han clasificado, según los distintos tipos de sistemas de representación simbólicos.

Las diferentes categorías establecidas permitieron el tratamiento de la información ofrecida en los textos de forma sistemática y organizada y por ello, la obtención de resultados que respondieran a los interrogantes planteados.

\section{Resultados}

La hermeneútica consistió en una explicación genética, en el sentido de señalar los sucesivos estadios de desarrollo en la utilización de los diferentes sistemas simbólicos en la enseñanza de los puntos críticos, intentando responder a la pregunta de cómo ocurrió dicha evolución mediante la enumeración de la secuencia genética de los diferentes sucesos. Es decir, se aporta una síntesis histórica de tipo genético, en el sentido de que se establece la secuencia cronológica y causal acerca de la enseñanza de los puntos críticos.

Para resumir y explicar todos los aspectos analizados en cada uno de los libros se desarrolla esta síntesis en tres apartados:

\section{Tabla 1. Tipos de análisis realizados}

\begin{tabular}{|l|l|}
\hline Análisis socio-cultural & $\begin{array}{l}\text { Explicación que justifique los contenidos y su } \\
\text { presentación en el libro en relación con la } \\
\text { época, el lugar y las circunstancias en que fue } \\
\text { escrito. }\end{array}$ \\
\hline Análisis epistemológico & $\begin{array}{l}\text { Analizar el status matemático que poseen los } \\
\text { contenidos del Análisis Matemático en cada } \\
\text { uno de los libros, concretamente en relación } \\
\text { con los puntos críticos. }\end{array}$ \\
\hline Análisis didáctico & $\begin{array}{l}\text { Ver el enfoque que reciben los puntos críti- } \\
\text { cos en cuanto a la enseñanza-aprendizaje ca- } \\
\text { racterizando los sistemas de representación } \\
\text { utilizados }\end{array}$ \\
\hline
\end{tabular}

Estos tres tipos de análisis nos muestran la evolución de la enseñanza del Análisis Matemático en los ciento cincuenta años que van desde su inicio 
hasta su consolidación, distinguiéndose, como se verá a continuación, tres periodos.

\section{Análisis socio-cultural}

Hay un primer periodo al que pertenecen los libros de l'Hôpital y MacLaurin caracterizados, desde el punto de vista de su estructura, por mantener la concepción clásica heredando la forma de hacer de los matemáticos griegos. A partir de unas definiciones iniciales en las que se establece el significado de los conceptos "primarios" que van a aparecer a lo largo del texto, se van a suceder las diferentes proposiciones que caracterizan las propiedades, estructura, reglas de cálculo en los que están involucrados dichos conceptos. Con cada una de dichas proposiciones se incluye un problema o ejercicio resuelto con la pretensión de ejemplificarla.

Un segundo periodo, corresponde a los libros de Euler y Lagrange que son más modernos en la organización de los contenidos, condicionados por la estructura algebraica de que se quiere dotar a los conceptos con el objetivo de que sea más coherente y consistente. Así se configura una moderna concepción del libro de texto matemático, que comienza con los fundamentos de la materia y culmina tras una exposición sistemática con las líneas más recientes de investigación.

Por todo ello el tipo de representación dominante es la representación simbólica: la ecuación algebraica o trascendente de la función que se quiere estudiar. Los problemas que se estudian tienen un carácter exclusivamente algebraico lo que supone una descontextualización de las funciones, ya que no se hace referencia ni a situaciones o formas de conocimiento que den lugar a ellas, ni siquiera a una descripción geométrica que permita su visualización.

Por último, están los libros de Bézout, Bails y Lacroix en los que se inicia un tratamiento de tipo didáctico característico de las primeras enciclopedias.

\section{Análisis epistemológico}

Inicialmente se posee una concepción geométrico-dinámica del Cálculo diferencial que se puede observar en el libro de L'Hôpital, pero sobre todo en el de MacLaurin. Las proposiciones y las demostraciones se llevan a cabo en un lenguaje puramente geométrico, y se realizan consideraciones acerca del cambio que se experimenta en el recorrido de las curvas: 
"Soit une ligne courbe MDM dont les apliquées PM, ED, PM soient paralleles entr'elles, \& qui soit telle que la couppée AP croissent continüellement, l'apliquée PM croisse aussi jusqu'à un certain point E, aprés lequel elle diminuë; ou au contraire qu'elle diminüe jusqu'à un certain point E, aprés lequel elle croisse. Cela posé, La ligne ED sera nommée La plus grande, ou la moindre apliquée. "(L'HÔPITAL, p. 41)

En un segundo periodo, se ensalzan los aspectos de tipo algebraico, tal es el caso de los libros de Euler y Lagrange. Uno de los cambios más importantes que se introdujo en el Análisis Matemático fue relativo a la noción de función. Hasta entonces había sido concebida como una relación entre dos variables, mientras que a partir de Euler empieza a pensarse como una asociación que a un número $\mathrm{x}$ le asocia otro $\mathrm{f}(\mathrm{x})$. Este cambio va a afectar al concepto de diferencial utilizado hasta entonces, ya que pasó de ser considerado como la variación de una variable, a la razón de cambio de la función en relación con el incremento de la variable.

Se realiza una revisión de la noción de diferencias finitas y la aplicación a las series para pasar a las diferencias infinitas, y las de cualquier orden. Se diferencia todo tipo de funciones desde las algebraicas a las trascendentes y las que tienen varias variables, deduciéndose las reglas y fórmulas para calcularlas para poder establecer una teoría de tipo general que permita diferenciar cualquier tipo de función según la expresión simbólica que tenga. En este sentido, las fórmulas pueden sustituir al concepto de diferencial, con lo que el Análisis Matemático se irá convirtiendo en algo puramente mecánico más que conceptual. Hay que destacar la utilización del cociente diferencial frente al diferencial que se había utilizado en el libro de L'Hôpital. Por ejemplo en el caso de los máximos y mínimos, se indica:

"Interim tamen ad casus, quibus valor ipsius y vel maximus vel minimus evadat, investigandos haec prima operatio instituenda es, ut differentiale functionis propositae nibilo aequetur atque ex aequtione $\frac{d y}{d x}=$ Oomnes ipsius $x$ valores eliciantur... Ostendemus enim fieri posse, ut neque maximum neque minum locum babeat sit $\frac{d y}{d x}$ " (EULER, p. 448)

Asimismo, hay que destacar el desarrollo en serie de potencias de las funciones que sugiere una dependencia de las expresiones analíticas y su estudio, con lo que se pierde el origen geométrico del Análisis, pasando a ocuparse más del estudio de las fórmulas.

Finalmente los libros de Bézout, Bails y Lacroix fijan su centro de interés en los métodos de cálculo de los puntos críticos, utilizando para ello el cociente diferencial aunque como cociente algebraico. 


\section{Análisis didáctico}

En el primer periodo, dado que no existe todavía un lenguaje funcional propio, ni siquiera la noción de función, todo el discurso descansa sobre el estudio y análisis de curvas de forma que las representaciones más utilizadas son las gráficas en las que las curvas mantienen su significado geométrico, de hecho, las curvas estudiadas son las clásicas como el Folium de Descartes o la parábola semicúbica de Neile. Los problemas que se tratan son exclusivamente geométricos y su estudio se hace desde este punto de vista, así los puntos críticos se clasifican atendiendo a este carácter, siendo el criterio la posición de la tangente: se distinguen puntos críticos que poseen tangente horizontal o vertical. Los términos utilizados para identificar los puntos de las curvas son diferentes de los actuales: coupée y apliquée.

En el libro de L'Hôpital se estudian de forma separada las curvas construidas a partir de un eje, de aquéllas cuyo elemento básico para su construcción es un único punto, desarrollándose de forma separada el método que permite calcular los puntos críticos en un caso o en otro, aunque en ambos se realiza a partir de lo que llama diferencia:

"La portion infiniment petite dont une quantité variable augmente ou diminue continuellement, en est appellée la Différence. "(L'HÔPITAL, p. 2)

En el libro de MacLaurin se hace el desarrollo en términos de las fluxiones de Newton

"On trouve les plus grandes \& les plus petites ordonnées, dans de pareils cas, (qui sont lesplus communs dans la résolution des Problêmes) en examinanten quel terme la Fluxión de la courbe devient égale à celle de la base, ou en quelque terme la Fluxión de l'ordonnée s'évanouit, celle de la base étant donnée. "(MACLAURIN, p. 156).

Sorprende la visión geométrica que poseen de las ecuaciones algebraicas o de las descripciones para poder realizar la construcción de curvas, bien a partir de otras

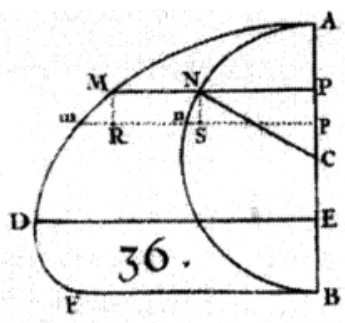

Soit une demiroulette acourcie AMF, dont la base BF est moindre que la demi-circonférence ANB du cercle générateur qui a pour centre le point C. Il faut déterminer le point E sur le diametre $\mathrm{AB}$, en sorte que l'apliquée ED soit la plus grande qu'il es posible. (L'Hôpital, p. 43) 
a partir de los datos de un problema

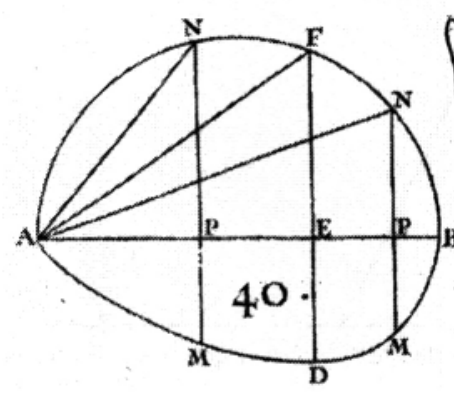

o a partir de un dibujo.

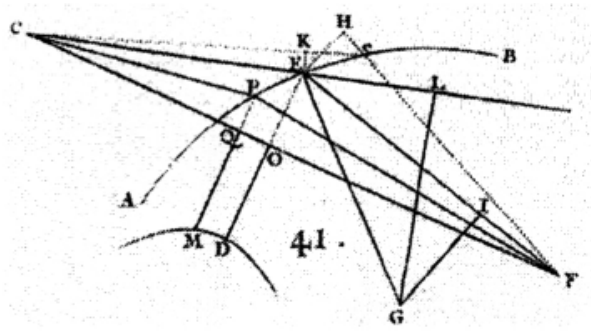

EXEMPLE VI. Entre tous les Cones qui peuvent éter inscrits dans une sphére, déterminer celuy qui a la plus grande surface convexe. (L'Hôpital, p. 46)

Hay que destacar las gráficas de MacLaurin en las que aparece al mismo tiempo, una curva y su curva derivada, lo que permite hacer comparaciones entre ambas, asociando las características gráficas de cada una de ellas, y la visualización de los diferentes tipos de puntos.

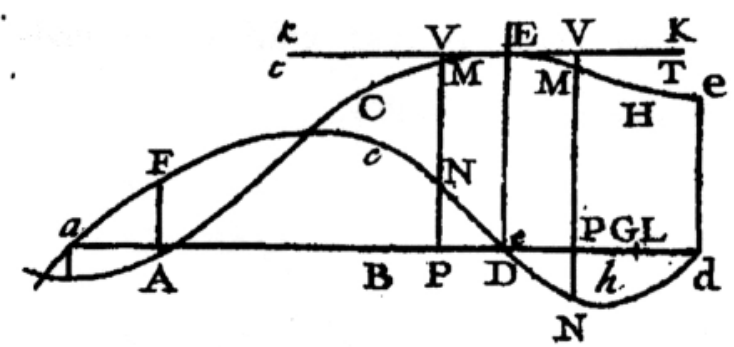

Las expresiones simbólicas que se utilizan en todos los textos representan toda una familia de funciones y se realiza un estudio de los puntos críticos en relación con los parámetros de dichas expresiones. Los tipos de 
funciones son muy variadas, y por ejemplo, en el de MacLaurin se manejan indistintamente las variables $\mathrm{x}$ e $\mathrm{y}$, haciéndose un estudio simétrico respecto de cada una de ellas.

En un segundo periodo, en los libros de Lagrange y Euler el tipo de representación predominante es la representación simbólica: la ecuación algebraica o trascendente de la función que se quiere estudiar.

Destaca el análisis detallado que hace Euler de las operaciones algebraicas de funciones y de su derivación, clasificando dichas funciones según su expresión simbólica y estudiando la diferenciación de cada uno de estos tipos de forma pormenorizada e independiente, estableciendo todas las posibilidades en relación con los parámetros que posean dichas expresiones simbólicas.

En el caso de Lagrange hay que mencionar su utilización de los desarrollos en serie de potencias sin necesidad de recurrir a las fluxiones, a las cantidades evanescentes o las diferenciales como habían hecho los matemáticos anteriores. A partir de la noción de función, realiza su desarrollo en serie, analiza la formación de los términos de dicha serie y establece la noción de función derivada. Esto determina que el Cálculo Diferencial se establezca a partir de la noción de función derivada y no en la noción de diferencial, como hemos visto en los otros libros escritos en Francia.

Finalmente, en el tercer periodo, en los libros de Bézout, Bails y Lacroix se utilizan los dos tipos de representación anteriores, intentando encontrar un equilibrio entre la mayor precisión de la representación algebraica con la visualización de las propiedades gráficas de la curva correspondiente.

Los problemas que se estudian son tanto aritméticos como geométricos y están planteados desde un punto de vista más didáctico que de la propia estructura de la matemática. Todos los problemas son genéricos en el sentido de que la solución corresponde a una familia de curvas, y muchos de ellos son de tipo demostración.

En estos libros se consideran indistintamente la variable x e y, así Bézout calcula los puntos críticos de una circunferencia:

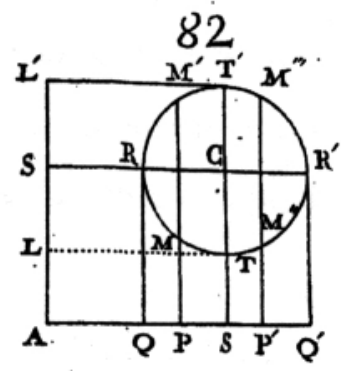


Y Lacroix representa gráficamente un mínimo con tangente vertical de la siguiente forma:

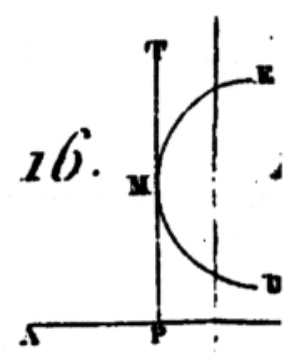

Se utilizan indistintamente representaciones gráficas y algebraicas buscando un equilibrio entre la mayor precisión de la segunda con la visualización de las propiedades de la gráfica de la curva correspondiente que proporciona la primera. La definición de puntos críticos es de tipo gráfico, asociando dichos puntos con un determinado comportamiento de la curva.

"La valeur qui a lieu dans le passage de l'acrroissement au décroissement, étant plus grande que celles qui la précèdent et qui la suivent immédiatement, s'appelle un maximum; le minimum es celle qui répond au point où le décroissement se change en accroissement ; celle-ci est par conséquent plus petite que les valeurs qui la précèdent et qui la suivent immédiatement : je dis immédiatement, parce qu'il y a des fonctions pour lesquelles ces alternatives ont lieu plusieurs fois. "(LACROIX, p. 96).

A partir de esta definición, se clasifican los distintos puntos de una forma gráfica: si tienen tangente horizontal o vertical, si cambian la convexidad de la curva, si dividen la curva en dos ramas,... Pero también se hace una clasificación de tipo algebraico. A partir de la ecuación de una curva se van analizando las diferentes posibilidades según la variación de los parámetros de la curva. Se trata, por lo tanto, de un estudio de tipo gráfico-analítico en el que el objetivo es la clasificación de los puntos críticos. Entre los distintos tipos de puntos se indica qué se entiende por punto singular:"On appelle points singuliers d'une courbe ceux dans lesquels elle offre quelque circonstance remarquable." (LACROIX, p. 96).

Y además de los máximos, mínimos y puntos de inflexión, se consideran puntos de retroceso de segunda especie en los cuales se forman dos ramas de la curva que tienen la misma tangente en dicho punto, de forma que ambas ramas permanecen en el mismo semiplano respecto de dicha recta tangente en un entorno del punto de retroceso. 


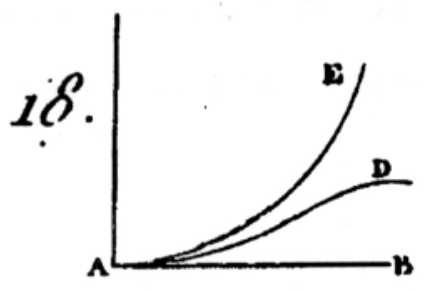

Los puntos múltiples:"Quelquefois les branches des courbes, au lieu de se réunir en se touchant, se coupent, et ont chacune leur tangente particulière." (LACROIX, p. 101)

Y por último, los puntos conjugados:

Les courbes sont accompagnées quelquefois de points isolés qui ont le caractère des points multiples; mais on les distingue, parce que les coefficients différentiels, y devenant imaginaires, soit dès le premier ordre, soit plus tard, montrent qu'il n'y a pas de points consécutifs (Lacroix, p. 103).

Todo ello configura, en este tercer periodo una generación de libros dedicados a la formación de profesionales que deben utilizar las matemáticas, caracterizados por su sentido didáctico.

\section{Consideraciones finales}

El análisis de las fuentes primarias y de los textos históricos nos sumergen en el mundo del matemático que vivió en una determinada época, nos da cuenta de las concepciones y la forma de pensamiento imperante en el momento, nos indica el tipo de problemas y las formas de solución que se gestaron según los instrumentos de los que se disponía, se recuperan algunas ramas de la matemática como, según hemos visto, la visión puramente geométrica de los conceptos propios del Análisis e incluso pueden ser fuente de actividades, situaciones o problemas para plantear a los alumnos y diseñar secuencias de aprendizaje, que proporciones una formación matemática más global y comprensiva que la que se ha practicado en los últimos años.

Entre las aportaciones que se han logrado con este estudio, se pueden destacar: la identificación de diferentes periodos en el tratamiento de los puntos críticos en los libros históricos de texto determinados por el momento sociocultural en el que fueron publicados, las concepciones que tenían los matemáticos en ese momento histórico y el tipo de representaciones que predominan en ellos, la determinación de las características de los sistemas sim- 
bólicos de representación utilizados en relación con los puntos críticos, la constatación de que el tipo de representación de los fenómenos relacionados con los puntos críticos favorecen la concepción de estos conceptos desde un punto de vista dinámico/estático según se enfatice la noción de variación asociada a las funciones o bien la estructura de la que forman parte estas funciones.

\section{Agradecimientos}

A Neuza Bertoni Pinto que realizó los contactos oportunos para que pudiera presentar este artículo para la revista Diálogo Educacional, por su ayuda para que se hiciera en tiempo y forma correctos y para que se ajustara a las normas de la revista.

A Ana Santiago de Coimbra (Portugal), una alumna del programa de doctorado de Educación Matemática de la Universidad de Salamanca y amiga que amablemente me tradujo al portugués el título y el resumen de este artículo.

\section{Referencias}

APOSTOL, Tom. Análisis matemático. Barcelona: Reverté, 1981.

BOYER, Carl. Historia de las matemáticas. Madrid: Alianza,1986.

BRADLEY, Gerald; SMITH, Karl. Cálculo de una variable. Madrid: Prentice may, 1998.

CANTORAL, Ricardo. Acerca de las contribuciones actuales de una didáctica de antaño: el caso de la serie de Taylor. Mathesis, Viseu, v.11, n. 1, p. 55-101, 1995

COHEN, Louis; MANION, Lawrence. Métodos de investigación educativa. Madrid: La Muralla, 1990.

CHEVALLARD, Yves. La transposition didactique du savoir savant au savoir enseigné. Grenoble: La Pensée Sauvage, 1985.

CHEVALLARD, Yves ; JOSHUA, M.A. Un exemple d'analyse de transposition didactique: la notion de distance. Recherche en Didactique des mathematiques. Grenoble, v.3, n.1, p.159-239, 1982.

CHOPPIN, Alain. L’histoire des manuels scolaires. Un bilan bibliomérique de la recherché français. Histoire de l'Education, Paris, n. 58, p. 165-185, 1993. 
DHOMBRES, Jean. French Mathematical Textbooks from Bézout to Cauchy. Historia scientarum, Tokio, n.28, p. 91-137, 1985.

DHOMBRES, Jean et al. Mathematiques au fil des äges. París: Gauthier-Vilars, [19-?].

DORMOLEN, Jean van. Textual Análisis. In: CHRISTIANSEN, Ben; HOWSON, Geoffrey; OTTE, Michael. (eds.) Perspectives on Mathematics Education. Dordrecht: Reidle, 1986. p. 141-171.

DUNHAM, William. Viaje a través de los genios. Madrid: Pirámide, 1993.

DURAN, José Antonio. Historia, con personajes de los conceptos del cálculo. Madrid: Alianza Universidad, 1996.

FOX, David Joseph. El proceso de investigación en educación. Pamplona: Universidad de Navarra, 1981.

GALL, Meredith; BORG; Walter; GALL, Joyce. Educational Research: an introduction. New York: Longman, 1996.

GONZÁlEZ, María Teresa. Sistemas simbólicos de representación en la enseñanza del Análisis Matemático: perspectiva histórica acerca de los puntos críticos. Tesis (doctoral) - Salamanca: Universidad de Salamanca, 2002 GRATTAN-GUINESS, Ivor Del cálculo a la teoría de conjuntos. Madrid: Alianza, 1984.

HAIRER, Ernest y WANNER, Gerhard. Analysis by its History. New York: Springer, 1996.

HOWSON, Geoffrey. Mathematics Textbooks: a comparative Study of grade 8 Texts. Vancouver: Pacific Educational,1995.

JANVIER, Claude. Problems of representation in the teaching and learning of mathematics. Londres: Lawrence Erlbaum, 1987.

LOWE, E. y PIMM, David. This is so: a text on texts. In: BISHOP, Alain et al. International Handbook of Mathematics Education. Dordrecht: Kluwer, 1996. p. 371-410.

OTTE, Michael. What is a text?. In: CHRISTIANSEN, Ben; HOWSON, Geoffrey; OTTE, Michael (eds.). Perspectives on mathematics education. Dordrecht: Reidel, 1986. p. 173-203.

PIMM, David. Speaking mathematically. New York Routledge y Kegan Paul. Traducción cast. (1990) El lenguaje matemático en el aula. Madrid: Ministerio de Educación y Ciencias-Ediciones Morata, 1987. 
PIMM, David. Mathematics classroom language form, function and force. In: BIELHER, Ronald et al. (eds.). Didactic of mathematics as a scientific discipline,.. Dordrecht: Kluwer. 1994. p.159-169.

SCHUBRING, Gert. On the methodology of analyzing Historical Textbooks: Lacroix as textbook author. For the learning of mathematics, Montreal, v.7, n.3, p. 41-51, 1987.

SIERRA, Modesto; GONZÁLEZ, María Teresa; LÓPEZ, Carmen. Una visión integradora acerca del concepto de límite. UNO. Revista de Didáctica de la Matemática. Barcelona, n.29, p. 77-94, 2002.

TAVIGNOT, Patricia. Analyse du processus de transposition didactique. Application a la symétrie orthogonale en sixieme lors la reforme de 1985. Recherches en didactique des mathematiques, Grenoble, v.13, n. 3, p. 257294, 1993.

VIÑAO, Antonio. De la importancia y utilidad de la historia de la educación (o la responsabilidad moral del historiador). En: GABRIEL, Narciso; VIÑAO, Antonio (eds.) La investigación histórico-educativa. Barcelona: Roncel, 1987.

WUSSING, Hans. Lecciones de Historia de las Matemáticas. Madrid: Siglo XXI, 1998. 